\title{
Patients and physicians' attitudes change on fecal microbiota transplantation for inflammatory bowel disease over the past 3 years
}

\author{
Yujie Zhang ${ }^{1,2 \#}$, Xianmin Xue ${ }^{2 \#}$, Song $\mathrm{Su}^{2 \#}$, He Zhou ${ }^{2}$, Yirong Jin ${ }^{2}$, Yanting Shi ${ }^{2}$, Junchao Lin ${ }^{2}$, \\ Jiayao Wang ${ }^{2}$, Xiaofei Li $^{2}$, Gang Yang ${ }^{2}$, Jessica R. Philpott ${ }^{3}$, Jie Liang ${ }^{2}$ \\ ${ }^{1}$ Department of Histology and Embryology, School of Basic Medicine, Xi'an Medical University, Xi'an, China; ${ }^{2}$ State Key Laboratory of Cancer \\ Biology, National Clinical Research Center for Digestive Diseases and Xijing Hospital of Digestive Diseases, Fourth Military Medical University (Air \\ Force Medical University), Xi'an, China; ${ }^{3}$ Department of Gastroenterology, Cleveland Clinic, Cleveland, OH, USA \\ Contributions: (I) Conception and design: J Liang, JR Philpott; (II) Administrative support: L Jie; (III) Provision of study materials or patients: L Jie; \\ (IV) Collection and assembly of data: Y Zhang, X Xue, S Su, H Zhou, Y Jin, Y Shi, J Lin, J Wang, X Li, G Yang; (V) Data analysis and interpretation: \\ J Liang, JR Philpott, Y Zhang, S Su; (VI) Manuscript writing: All authors; (VII) Final approval of manuscript: All authors. \\ \#These authors contributed equally to this work. \\ Correspondence to: Jie Liang. State Key Laboratory of Cancer Biology, National Clinical Research Center for Digestive Diseases and Xijing \\ Hospital of Digestive Diseases, Fourth Military Medical University (Air Force Medical University), 127 Changle West Road, Xi'an 710032, China. \\ Email: liangjiel@fmmu.edu.cn; Jessica R. Philpott. Department of Gastroenterology, Cleveland Clinic, 9500 Euclid Ave A30, Cleveland, OH 44195, \\ USA. Email: philpoj@ccf.org.
}

Background: In the past 3 years, increasing data and experience has become available regarding fecal microbiota transplantation (FMT) for the treatment of inflammatory bowel disease (IBD). However, how this increase in knowledge has impacted the attitudes of patients and physicians is largely unknown. This study aimed to investigate the change of patients' and physicians' attitudes towards FMT for IBD treatment. Methods: Questionnaires for patient and physician attitude on FMT for IBD were pilot-tested and developed. Patients and physicians from the same groups completed the questionnaires in 2016 and 2019, separately. The attitudes towards efficacy, adverse events, and methodological features of FMT in 2016 were compared with those in 2019.

Results: A total of 1,255 questionnaires from 486 patients and 769 physicians were collected. Over the 3 years, an increased number of patients had heard of FMT and had similarly positive opinions towards using FMT for IBD therapy. Additionally, patients retained the tendency to overestimate the efficacy. The physicians' perceptions became closer to the findings reported in recent studies in 2019 compared with 2016. However, only a minority of patients and physicians understood the frequency required of FMT courses for induction of clinical remission. In particular, both patients and physicians underestimated the risk of mild adverse events and IBD flare.

Conclusions: Patients are receptive towards FMT as therapy for IBD but opportunity remains to improve understanding of benefit and potential risks. Physicians also demonstrated knowledge gaps in use of this therapy. Aligning patient preference and physician knowledge gap will lead to better education and facilitate the development of decision-making guidelines.

Keywords: Inflammatory bowel disease (IBD); fecal microbiota transplantation (FMT); IBD flare; medical communication and education

Submitted Feb 26, 2021. Accepted for publication Oct 13, 2021.

doi: $10.21037 /$ atm-21-3683

View this article at: https://dx.doi.org/10.21037/atm-21-3683 


\section{Introduction}

Fecal microbiota transplantation (FMT) is a growing and promising therapy in which fecal microbacteria from a healthy donor is transferred to the gastrointestinal tract (GIT) of a patient $(1,2)$. In the recent past, it was first widely reported for the treatment of Clostridium difficile infection (CDI) and has been shown to be extremely effective $(3,4)$. The FMT has also been reported in the treatment of several kinds of diseases, including obesity (5-7), metabolism symptoms $(6,8)$, diabetes, autism (9), and so on, with equivocal results. The attitudes of patients and physicians have been investigated in some studies, but the focus has been on aspects such as the role of FMT in the treatment of CDI and route for the procedure (10-12). Whether the perceptions were accurate based on current bulk evidence on risks and benefits, was not reported.

The treatment of inflammatory bowel disease (IBD) has rapidly evolved in recent years and FMT is considered to have potential roles in treating IBD (13). Promising case reports, observational cohort studies, and even randomized controlled trials (RCTs) have been published on the treatment of IBD with FMT (14). However, FMT for IBD treatment has remained controversial, largely due to inadequate and conflicting evidence regarding its safety and efficacy. As an example, 2 RCT studies reported conflicting results on FMT for the treatment of ulcerative colitis (UC) in $2015(15,16)$. To broaden the research regarding patient attitudes, 2 studies were recently published, and patients were reported to be interested in FMT as a potential therapy $(17,18)$. Our study had similar findings regarding patient attitudes (unpublished data in 2016). Two studies about attitude toward FMT were published in recent years. One was focus on the attitude changes after FMT, and the other was about patient attitudes toward the use of FMT in the treatment of recurrent CDI $(19,20)$. None of two studies focus on the change of attitudes in patients and physicians over time. As more studies have been performed on FMT in the treatment of IBD in the past 3 years, including well-designed RCTs published on high impactfactor journals $(21,22)$, and new recommendations have been developed (23-25). These data might have changed patients' and physicians' attitudes, but this change is still unknown. In order to discover the impact, this study was conducted to compare patients' and physicians' attitudes in 2019 with those in 2016. We present the following article in accordance with the SURGE reporting checklist (available at https://dx.doi.org/10.21037/atm-21-3683).

\section{Methods}

In 2016, questionnaires for both patients and physicians regarding the risks and benefits of FMT for IBD were developed and pilot-tested in a sample of respondents. The patient respondents were samples of IBD patients in the clinic of IBD center affiliated to the Digestive Disease Department of Xijing Hospital. The physician respondents were sampled from other tertiary hospitals including the Second Affiliated Hospital to Jiao Tong University in Xi'an, the Second Affiliated Hospital to Airforce Medical University, and General Hospital of Ningxia Medical University (all institutions could perform FMT for IBD treatment). The development process included face-toface interviews to ensure survey comprehension. After an iterative review process, the questionnaires were finalized. Questions regarded awareness of FMT, estimation of the remission rate as a result of FMT at the end of 2 months, times needed for induction of clinical remission, incidence of adverse effects, preference of transplantation route, and gender, age, and education of the respondents. Translated questionnaires for patients and physicians have been attached as Supplementary files S1,S2, respectively. Next, the questionnaires were distributed to adult patients at the clinic of IBD center affiliated to the Digestive Disease Department of Xijing Hospital and physicians at nation-wide IBD conferences, respectively. All patients were told that it was totally acceptable if they were unwilling to participate in this investigation and the questionnaires would not collect their individual private data, thus the questionnaires were completed. If a patient had never heard of FMT, a brief introduction of FMT was given as following: "FMT is a novel treatment for IBD. In this treatment, the microbiome extracted from a bealthy donor will be delivered to the gut of the patient by variable means. It can improve or even cure the gut inflammation by restoring or improving the residual gut microbiota. This therapy is currently under research and mostly performed for IBD patients who are refractory to conventional treatment. Though promising results have been frequently reported, more trials are needed." Finally, all completed questionnaires were collected and primarily analyzed by SS and XXM. No incentives were provided.

Thereafter in 2019, the same questionnaires were distributed to patients from Xijing IBD center and physicians from similar level IBD conferences (XXM and ZYJ). There was overlap in that some patients and physicians completed the survey in both 2016 and 2019. The results were analyzed and compared by 
2 researchers (SS and XXM). The 2 researchers worked independently in order to increase inter-coder reliability. If a disagreement occurred, they discussed with a third researcher (LJ) until a consensus was achieved. The study was conducted in accordance with the Declaration of Helsinki (as revised in 2013). The study was approved by the Chinese Ethics Committee of Registering Clinical Trials (ChiECRCT20200202) and informed consent was taken from all the subjects.

\section{Statistical analysis}

Simple size calculation and analyses of the data were conducted using SPSS statistical software, version 21 (IBM Corp., Chicago, IL, USA). Descriptive statistics were performed to report the results. Categorical data were expressed as percentages, and quantitative data were expressed as median. Mann-Whitney $\mathrm{U}$ test, Chi-square $\left(\chi^{2}\right)$ test, or Fisher's exact test were used to compare differences between 2016 and 2019 where appropriate. Ordinal survey items were compared between more than 2 groups using the Kruskal-Wallis test and between 2 groups using the Wilcoxon rank sum test. Comparison of the nonordinal data were conducted using the $\chi^{2}$ test. A P value $<0.05$ (2-sided) was deemed significant.

\section{Results}

A total of 534 (316 in 2016 and 218 in 2019) questionnaires were distributed and 486 (282 in 2016 and 204 in 2019) questionnaires were completed in patients, with a response rate of $89.2 \%$ in 2016 and $93.6 \%$ in 2019 , respectively (Table 1). In 2016, 84.3\% of respondents had UC and $15.7 \%$ had Crohn's disease (CD). The median duration of disease was 3 years. It was found that $4.6 \%$ of the respondents received infliximab treatment and $12.1 \%$ received immunosuppressive medications. In 2019, the percentage of $\mathrm{CD}$ respondents increased to $29.9 \%(\mathrm{P}=0.047)$. The median duration of disease was 4 years. Infliximab treatment was undertaken by $15.2 \%$ of the patients $(\mathrm{P}=0.045)$ and $21.1 \%$ received immunosuppressive medications $(\mathrm{P}=0.039)$. Moreover, age distribution, self-perceived disease activity, levels of education, and previous/current treatment are also summarized in Table 1 . In addition, $40.7 \%$ of respondents had heard of FMT in 2016, while this number increased to $56.4 \%$ in $2019(\mathrm{P}=0.024)$. The number of patients willing to undergo FMT was comparable between 2016 and 2019 $(60.6 \%$ vs. 62.7\%) (Table 1$)$.
A total of 1,050 (500 in 2016 and 550 in 2019) questionnaires were issued and 769 (389 in 2016 and 380 in 2019) physicians completed the questionnaires, with a response rate of $77.8 \%$ in 2016 and $69.1 \%$ in 2019 , respectively (Table 2). Most of the physician respondents were from tertiary hospitals $(55.8 \%$ in 2016 and $60.3 \%$ in 2019). Gastroenterologists accounted for the majority of respondents (62.2\% in 2016 and $75.8 \%$ in 2019). The remaining portion of physician respondents were internal medical doctors. Meanwhile, most of the physicians (59.1\% in 2016 and $61.9 \%$ in 2019 ) had a postgraduate degree. An increase from $67.1 \%$ in 2016 to $97.3 \%$ in 2019 of physicians had heard of FMT $(\mathrm{P}=0.017)$. Additionally, more physicians were willing to recommend FMT to patients with CDI $(77.1 \%)(\mathrm{P}=0.031)$ or $\mathrm{UC}(73.9 \%)(\mathrm{P}=0.046)$ in 2019. Regarding CD, IBS, constipation, and several other diseases, the percentage of doctors who would recommend this treatment remained similar between 2016 and 2019.

\section{Clinical remission}

In 2016, $53.2 \%$ of the UC patients estimated $51-70 \%$ patients receiving FMT would achieve 2-month clinical remission. Patients were not so optimistic in 2019 (47.1\%), but the difference was not significant $(\mathrm{P}=0.506)$ (Figure 1A). These findings were similar to those among patients with CD (Figure 1B) $(\mathrm{P}=0.781)$.

Most physicians believed $20-50 \%$ of UC patients could achieve clinical remission in 2 months after FMT, which increased from $41.4 \%$ in 2016 to $52.9 \%$ in $2019(\mathrm{P}=0.031)$ (Figure 1C). Regarding CD, the percentage of physicians who believed $20-50 \%$ of patients could achieve clinical remission in 2 months after FMT decreased from 51.4\% to $46.6 \%$, but with no statistical significance $(\mathrm{P}=0.102)$ (Figure 1D).

\section{Adverse events}

Estimated risk of mild adverse events of patients' perception are shown in Figure 2A: $40.8 \%$ in 2016 and $54.9 \%$ in 2019 believed $20-50 \%$ of patients would suffer mild adverse events after/during FMT $(\mathrm{P}=0.045)$. The estimated chance for IBD flares was considered to be less than $10 \%$ in $83.3 \%$ of patients in 2016 and $80.9 \%$ in $2019(\mathrm{P}=0.539)$ (Figure $2 B$ ).

Risk of mild adverse events after/during FMT was mostly estimated as $20-50 \%$ by $46.8 \%$ of physicians in 2016 and $56.8 \%$ in $2019(\mathrm{P}=0.027)$ (Figure $2 C$ ). Less physicians estimated $<10 \%$ risk of IBD flare after the FMT in 2019 (45.0\%) compared to that in $2016(73.8 \%)$ 
Table 1 Patient respondent characteristics

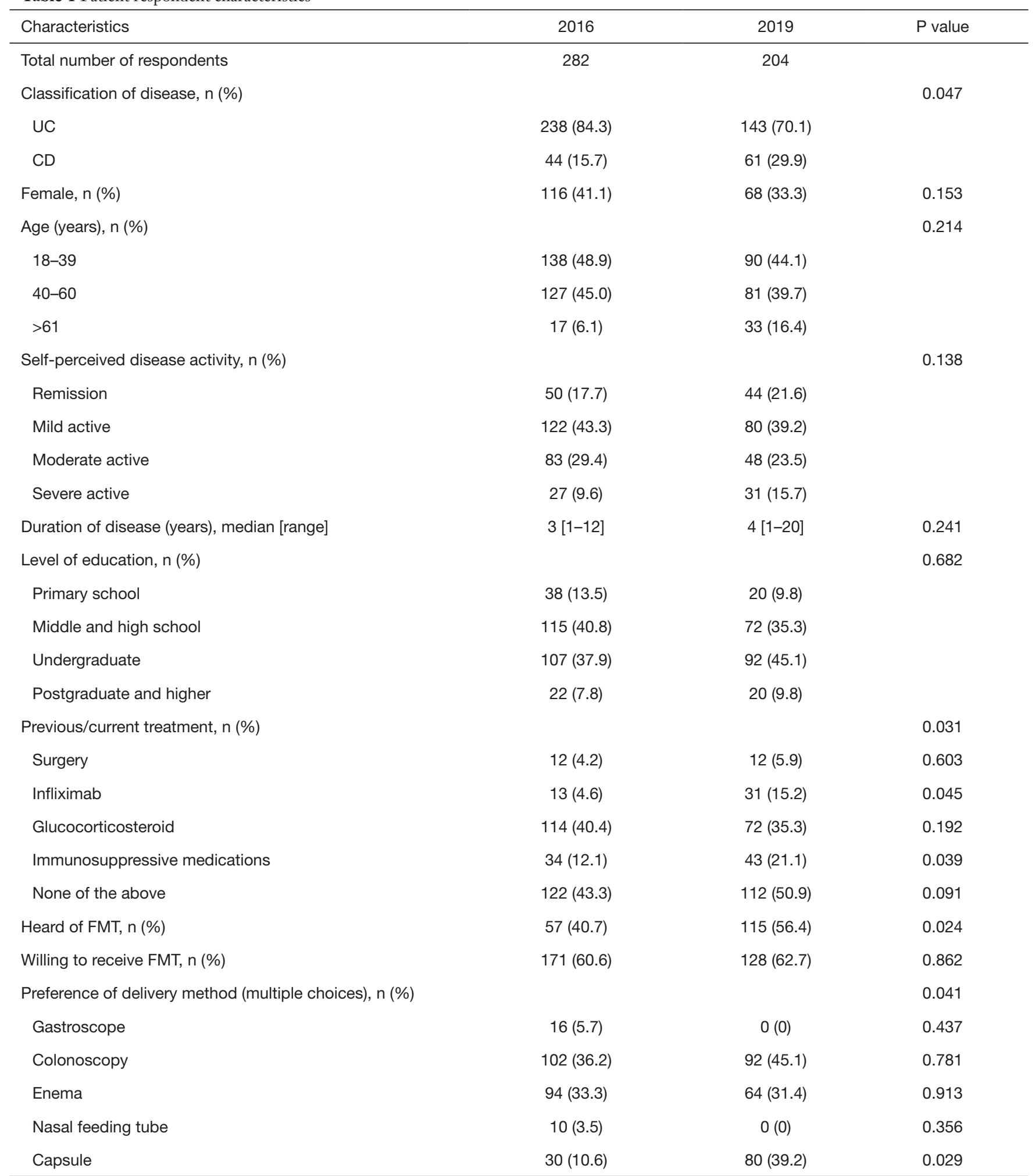

UC, ulcerative colitis; CD, Crohn's disease; FMT, fecal microbiota transplantation. 
Table 2 Physician respondent characteristics

\begin{tabular}{|c|c|c|c|}
\hline Characteristics & 2016 & 2019 & $P$ value \\
\hline \multicolumn{3}{|l|}{ Level of hospital, n (\%) } & 0.104 \\
\hline Tertiary & $217(55.8)$ & $229(60.3)$ & \\
\hline Secondary & $114(29.3)$ & $120(31.6)$ & \\
\hline \multicolumn{3}{|l|}{ Subspecialty, n (\%) } & 0.069 \\
\hline Gastroenterologist & $242(62.2)$ & $288(75.8)$ & \\
\hline Physicians from internal medicine & $70(18.0)$ & $47(12.4)$ & \\
\hline Others & $77(19.8)$ & $45(11.8)$ & \\
\hline Postgraduate & $230(59.1)$ & $235(61.9)$ & \\
\hline $\mathrm{PhD} / \mathrm{MD}$ & $36(10.3)$ & $61(16.0)$ & \\
\hline \multicolumn{3}{|l|}{ Level of the physicians, $n$ (\%) } & 0.052 \\
\hline Professors & $91(23.4)$ & $160(42.1)$ & \\
\hline Attendings & $93(23.9)$ & $60(15.8)$ & \\
\hline Residents and under & $205(52.7)$ & $160(42.1)$ & \\
\hline Heard of FMT, n (\%) & $261(67.1)$ & $370(97.3)$ & 0.017 \\
\hline \multicolumn{3}{|c|}{ Willing to recommend FMT for different disease (multiple choices), n (\%) } & 0.024 \\
\hline Diabetes & $47(12.1)$ & $53(13.9)$ & 0.911 \\
\hline Metabolic syndrome & $56(14.3)$ & $82(21.6)$ & 0.585 \\
\hline Autism & $23(6.0)$ & $50(13.2)$ & 0.118 \\
\hline \multicolumn{3}{|c|}{ Preference of delivery method (single choice), $n(\%)$} & 0.045 \\
\hline Gastroscope & $33(8.5)$ & $10(2.6)$ & 0.185 \\
\hline Colonoscopy & $142(36.5)$ & $174(45.8)$ & 0.167 \\
\hline Enema & $140(35.9)$ & $90(23.7)$ & 0.202 \\
\hline Nasal feeding tube & $38(9.8)$ & $0(0)$ & 0.157 \\
\hline Capsule & $36(9.3)$ & $106(27.9)$ & 0.032 \\
\hline
\end{tabular}

FMT, fecal microbiota transplantation; CDI, clostridium difficile infection; UC, ulcerative colitis; CD, Crohn's disease; IBS, irritable bowel syndrome; FMT, fecal microbiota transplantation. 
A UC-2-month efficacy-patients 2016

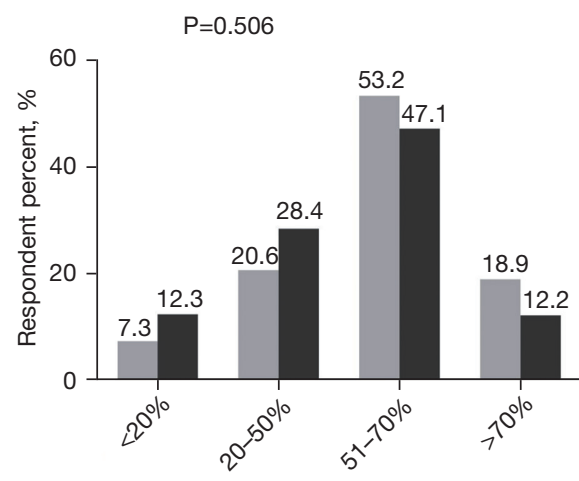

C UC-2-month efficacy-physicians $\mathrm{P}=0.031$

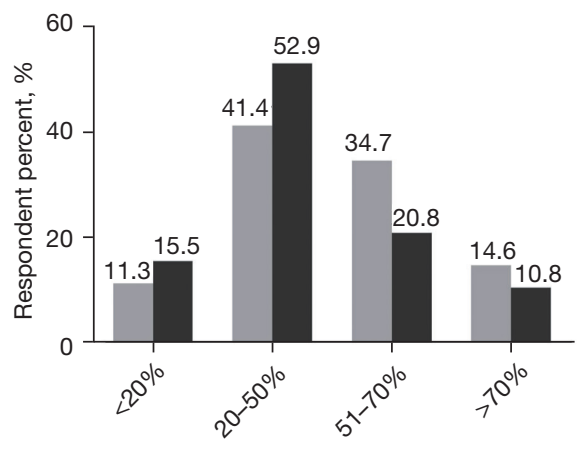

B CD-2-month efficacy-patients
2019

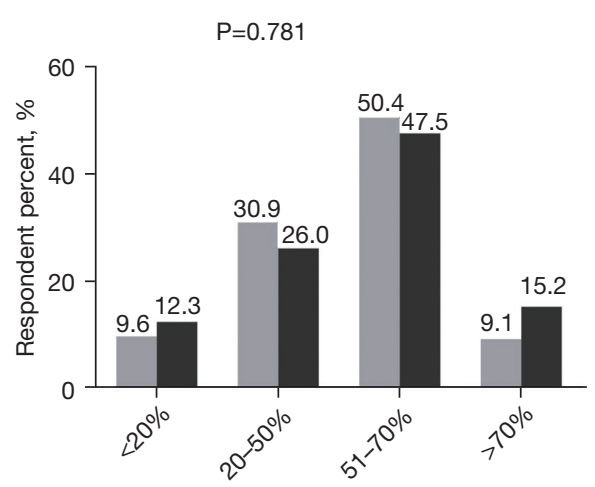

D CD-2-month efficacy-physicians

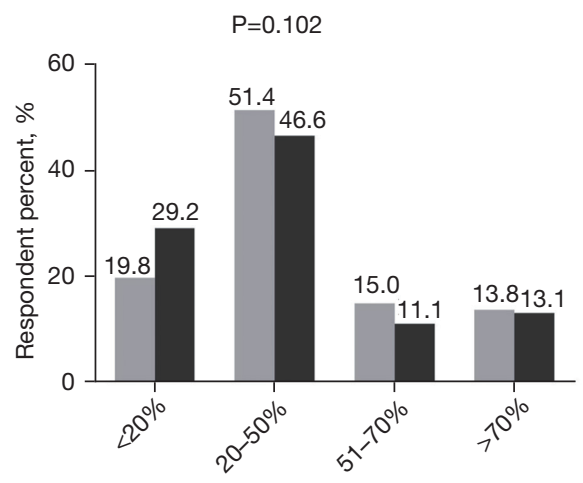

Figure 1 The estimated chance of clinical remission in 2 months after FMT. (A) UC patients' perception; (B) CD patients' perception; (C) physicians' perception towards UC; (D) physicians' perception towards CD. $\mathrm{P}$ value represents comparison between data in 2016 and that in 2019. FMT, fecal microbiota transplantation; UC, ulcerative colitis; CD, Crohn's disease.

$(\mathrm{P}=0.011)$ (Figure 2D).

\section{Methodological features}

Regarding the times needed for induction of clinical remission, most patients $(90.6 \%$ in 2016 and $84.3 \%$ in 2019) presumed 1-5 times was adequate $(\mathrm{P}=0.057)$ (Figure $3 A)$. Lastly, regarding the route of administration, most patients indicated preferences for colonoscopy (36.2\%) and enema (33.3\%) in 2016, while in 2019, patients were more likely to preference colonoscopy (45.1\%) and capsule (39.2\%). It was an especially large increase from $10.6 \%$ to $39.2 \%$ of preference for FMT through capsule $(\mathrm{P}<0.001)$ (Figure $3 B$ ).

Only $5.9 \%$ of the physicians in 2016 believed more than 5 repeats of FMT were needed to induce clinical remission of IBD, while in 2019 this number increased to $18.7 \%(\mathrm{P}<0.001)$ (Figure $3 C)$. With regard to the preferred administration route, most physicians preferred colonoscopy $(36.5 \%)$ and enema $(35.9 \%)$ in 2016 , which changed to colonoscopy (45.8\%), capsule (27.9\%), and then enema (23.7\%) $(\mathrm{P}=0.034)$ in 2019 (Figure 3D).

\section{Discussion}

In our study, we found that patients retained the tendency to overestimate the efficacy of FMT in IBD. The physicians' perceptions became closer to the findings reported in recent studies in 2019 compared with 2016. However, only a minority of patients and physicians understood the frequency required of FMT courses for induction of clinical remission. In particular, both patients and physicians underestimated the risk of mild adverse events and IBD flare. 
A

Mild adverse events-patients

$\mathrm{P}=0.045$

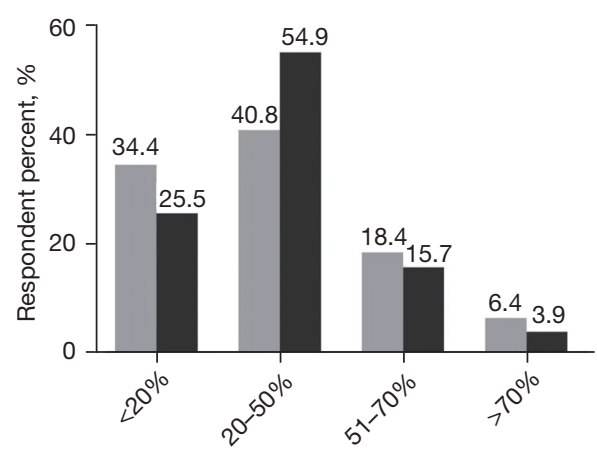

C Mild adverse events-physicians

$\mathrm{P}=0.027$

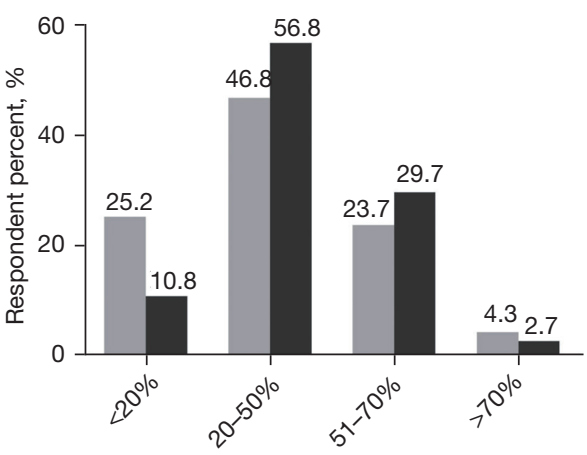

\section{B}

$2019 \quad$ IBD flare-patients

$\mathrm{P}=0.539$

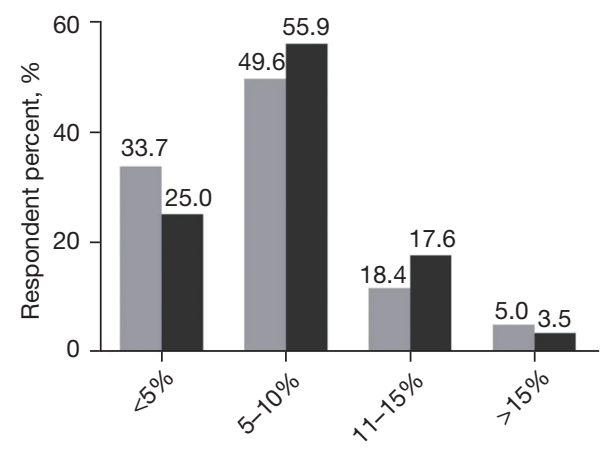

D IBD flare-physicians

$\mathrm{P}=0.011$

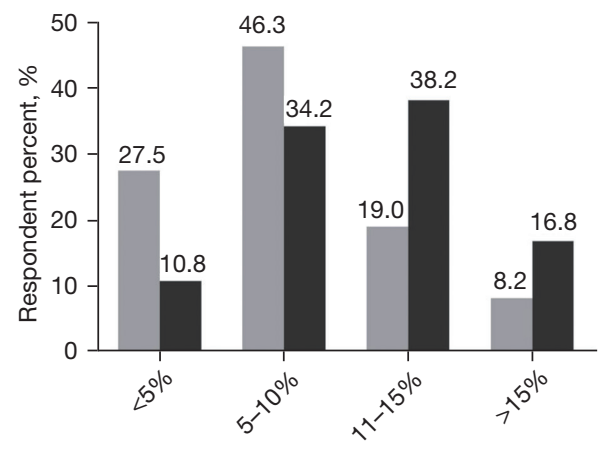

Figure 2 The perceptions towards adverse events of FMT. (A) Patients' perception towards mild adverse events; (B) patients' perception towards the risk of IBD flares; (C) physicians' perception towards mild adverse events; (D) physicians' perception towards the risk of IBD flares. $\mathrm{P}$ value represents comparison between data in 2016 and that in 2019. FMT, fecal microbiota transplantation; IBD, irritable bowel disease.

There is promise for FMT as a potentially powerful treatment for patients with IBD. As with all medical procedures, it is very important for both the patients and the physicians to fully understand the risks and benefits of FMT. Since each patient has a different threshold for acceptable risks or benefits, individual patient preferences need to be included in the decision-making process. Before patients can be engaged in this process, they first need to understand the benefits and risks of FMT. There have not yet been any studies investigating patients' and physicians' understanding of the efficiency and possible risks of FMT for IBD.

The dysbiosis of gut microbiota plays an important role in the pathogenesis of IBD. The change of microbiota triggered an aberrant mucosal immune response and then leaded to the development of chronic intestinal inflammation in IBD patients (24). The diversity of microbiota was decrease in IBD patients, including a decrease in Clostridium and a subsequent increase in Enterobacteriaceae (25). FMT could restore the normal gut microbiota and relieve the patient from pathological symptoms (26). This leads to an increased interesting in FMT in the patients and physicians. In this study, the differences in questionnaire results between 2016 and 2019 suggested more patients had heard of FMT and retained enthusiasm for FMT, around $60 \%$ of the patients were willing to accept FMT as a treatment for their diseases. However, most patients overestimated the efficacy of FMT. As for the physicians, up to $97.3 \%$ of the 
A Infusion numbers needed-patients 2016

$\mathrm{P}=0.057$

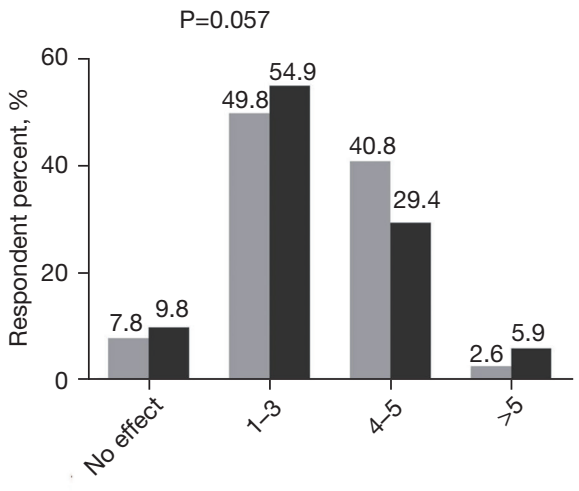

C Infusion numbers needed-physicians

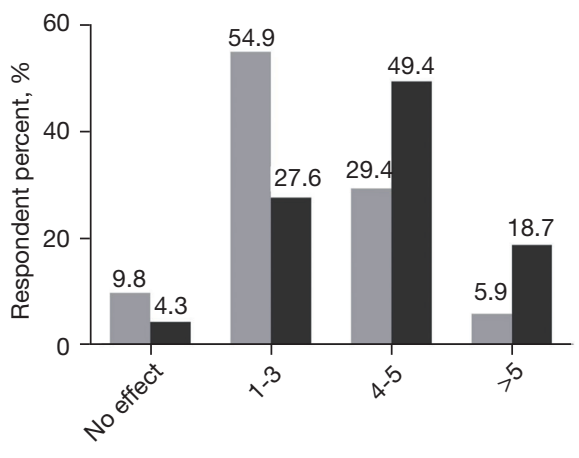

B Administration route-patients 2019

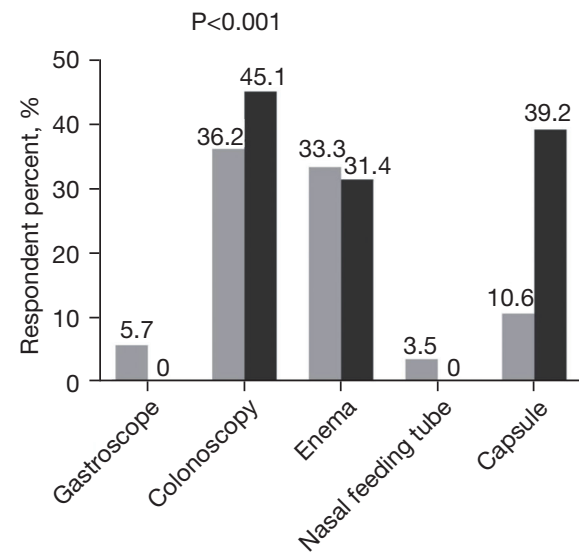

D Administration route-physicians $\mathrm{P}=0.034$

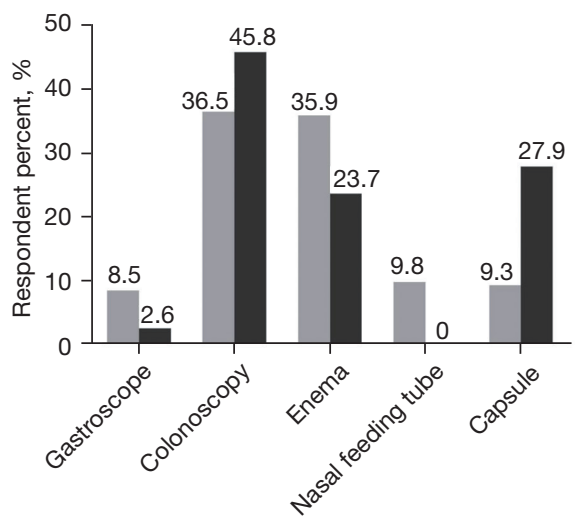

Figure 3 The perceptions towards methodological features of FMT. (A) Patients' perception towards the estimated numbers needed of FMT infusion for induction of a clinical remission; (B) patients' preference (multiple choices) of FMT administration route; (C) physicians' perception towards the estimated numbers needed of FMT infusion for induction of a clinical remission; (D) physicians' preference (single choice) of FMT administration route. P value represents comparison between data in 2016 and that in 2019. FMT, fecal microbiota transplantation.

respondents had heard of FMT and most of the physicians had achieved rational perceptions on the efficacy of FMT. Notably, both physician and patient respondents might have underestimated the number of treatments needed for induction of 2-month clinical remission and the risk of common mild adverse events and flares.

There was no previous data about the exact percentage of patients who had heard of FMT, especially to compare if it had increased with more information having been provided. In this study, $40.7 \%$ of the patient respondents had heard of FMT in 2016. This number increased to $56.4 \%$ over 3 years, potentially on account of proper patient education and the spread out of popular medical information online (27). Zipursky et al. also explored the attitude of patients towards using FMT in the treatment of CDI and confirmed that patients are open to FMT as an alternative treatment for recurrent CDI (20). The percentage of physicians who had an awareness of FMT has increased from $67.1 \%$ in 2016 to $97.3 \%$ in 2019 in this study. In another study, researchers revealed a low recognition 
level of postgraduate medical students for FMT (28). Considering that in this study most of the respondents were gastroenterologists, a greater awareness of FMT was reported in our study.

For patients and providers to have an effective discussion of FMT as therapy for IBD, a clear understanding of the currently available data is important. A recent Cochrane systematic review provided the data that $37 \%$ of the UC patients who received FMT as treatment achieved clinical remission at week 8 , but the quality of data was low and further studies are required (14). As for CD patients, another systematic review and meta-analysis pooled data from 11 studies (4 case reports and 7 prospective uncontrolled cohort studies including $83 \mathrm{CD}$ patients) on FMT for CD and reported that $50.5 \%(42 / 83)$ of $\mathrm{CD}$ patients achieved clinical remission (29), although we believe that controlled studies are required to better investigate the role of FMT. In our study, $53.2 \%$ of patients in 2016 and $47.1 \%$ in 2019 estimated $50-70 \%$ efficacy for UC. Whereas, the majority (41.4\% in 2016 and $52.9 \%$ in 2019 ) of physicians presumed 20-50\% efficacy for UC, which is consistent with the realworld data. Conversely, 51.4\% of physicians in 2016 and $46.6 \%$ in 2019 estimated $20-50 \%$ efficacy of FMT for CD. In addition, we revealed that $46.5 \%$ of physicians in 2016 and $47.4 \%$ in 2019 would recommend that CD patients undergo FMT treatment and this feature can be explained by the following reasons: firstly, the perception of several physicians may be influenced by previous observational studies such as the above systematic review and metaanalysis reporting a CD clinical remission rate of 52-76.7\% post initial FMT treatment (29-32); secondly, the high clinical remission rate of CD after FMT treatment actually remains controversial in daily clinical practice, because there were no RCTs or non-randomized studies with a control arm eligible for inclusion and analysis in the systematic review and meta-analysis above and all the previous studies were observational studies with no controls, such as case reports and uncontrolled cohort studies. Additionally, there was no study of mucosal healing and a publication bias was reported. Mucosal healing is important in particular for studies of CD due to lack of correlation between symptoms and mucosal healing. Thus, physicians who recommended that CD patients undergo FMT remain a minority. Taken together, the data above suggested that patients might overestimate the efficacy, while the physicians are more rational. It would be helpful for the physicians communicate more with their patients, who would then become more objective towards the treatment.
A full understanding of the most effective application of FMT, including number of treatments required, method to keep FMT effective, and duration remains unclear based on current literature (33). FMT for delivery of treatment have involved the upper gut through oral intake of fecal microbiota capsules, middle gut through endoscopy, nasojejunal tube, and percutaneous endoscopic gastrostomy with jejunal extension, and lower gut through colonoscopy, enema, distal ileum stroma, colostomy, and colonic transendoscopic enteral tubing (34). The methods to keep the survival of microbiota after transplantation were shown as follows, (I) shortening the preparation time and air exposure time of fecal microbiota; (II) using fresh stool evacuated within the preceding $6 \mathrm{~h}$; and (III) cooling the specimen in $2-8{ }^{\circ} \mathrm{C}$ until application (35). Rossen et al. administered FMT via a nasoduodenal tube and achieved $30.4 \%$ clinical remission within 3 weeks (15). Moayyedi et al. performed FMT enemas weekly for 6 weeks found that 9 of 38 (24\%) in the FMT group achieved remission (16). Paramsothy et al. administered FMT by colonoscopy 5 times per week for 8 weeks, and $27 \%$ experienced steroidfree clinical remission with endoscopic remission or response (21). In a parallel trial, Costello et al. administered FMT via colonoscopy and 2 additional enemas over a 7-day period and achieved remission in $32 \%$ of patients (22). A recent systematic review and meta-analysis confirmed that UC patients receiving $>10$ administrations of FMT achieve a considerably higher rate of clinical remission (49\%) than that in patients who received $\leq 10$ infusions (27\%) (36). Considering the above studies, both patients and physicians might underestimate the duration or number of therapies required to achieve successful therapy. In the present study, less than $10 \%$ of patients and $20 \%$ of physicians predicted more than 5 times of FMT were needed for clinical remission.

The FMT therapy is not risk free, and mild and less commonly serious events have been reported. Adverse event rates were similar in the FMT group and the control group (14). The most common adverse events are mild ones, including abdomen discomfort, abdomen pain/cramping, bloating, diarrhea, nausea, borborygmus, headache, dizziness, and/or fever $(14,36)$. Severe complications are rare and mainly consist of the following types: infection, IBD flares, aspiration, and death. According to previous studies, common adverse events and the frequency of IBD flares associated with FMT are about $78 \%$ and $14.9 \%$ $(14,15,21,36)$, respectively. In our study, $40.8 \%$ of patients in 2016 and $54.9 \%$ of patients in 2019 predicted that 
$20-50 \%$ of FMT patients would have mild adverse events. This estimate was similar for physicians (46.8\% in 2016 and $56.8 \%$ in 2019). Both the patients' and physicians' estimation of mild adverse events was lower than the results reported in recently published articles, which reported around that $13 \%$ suffer an IBD flare after FMT (37). In our study, $66.3 \%$ of patients in 2016 and $75.0 \%$ in 2019 estimated $>5 \%$ chance of IBD flare after FMT. The number of physicians predicting an accurate rate of IBD flares increased from $72.5 \%$ to $89.2 \%$ from 2016 to 2019 , which suggested the physicians had become more aware of the risk of adverse events of IBD flare.

The questionnaire attempted to elucidate patient preferences as to route of administration. A multiple-choice question was used to assess this concern. Interestingly, an increasing preference for the capsule transplantation route was found in patients, partially because of the widespread information of capsule-delivered FMT. These data will potentially be used to support further studies on FMT transplant route. The physicians' recommendation might have a certain effect on patient preferences. Both the data in 2016 (36.5\% of respondents) and 2019 (45.8\% of respondents) suggested colonoscopy was the most preferred method of transplantation.

This study had several strengths. First, the major strength of this study was that the change of attitude of patients and physicians over time was reported. This was the only study of which we are aware that assessed the change in attitudes in a similar group of people over time. This study also included both patients and physicians, allowing a more comprehensive understanding of the attitudes of these 2 groups who participate in decision making for IBD care. Secondly, most of the questions in this study were quantitative and the results were compared with latest or comprehensive research data. Third, various methods were used to ensure the quality of the questionnaire, including explaining the content and how to fill the questionnaire to the patients and physicians carefully before answering the questionnaire, and using the face-toface interviews to ensure survey comprehension. Finally, this was the first survey not only on the willingness, but physician and patient understanding, of estimated efficacy and route, the time needed for clinical remission, risks of mild adverse events, and IBD flares of FMT for IBD. There were also some limitations to this study. Firstly, similar to previous studies, the questions were hypothetically designed and patients filled out the questionnaire anonymously; thus, in real life, patients' decision making about possible
FMT may be different. Next, the exact percentage of patients and physicians who took both tests in 2016 and 2019 were unclear, as the questionnaires were completed anonymously; however, based on the patients' data of our IBD cohort and knowledge of our faculty, we suppose the overlapped percentage may be $70 \%$ in patient respondents and $50 \%$ in physician respondents, respectively. Finally, the people surveyed were from one hospital in China, and understanding if these trends in attitudes towards FMT as a therapy for IBD are also held in other parts of the country and world will be of interest for the future.

\section{Conclusions}

As more IBD therapies are developed and data regarding efficacy and risks collected, the community of IBD providers will work to develop pathways to position therapies and provide decision making guidelines [such as American Gastroenterological Association (AGA), European Crohn's and Colitis Organization (ECCO), and the Chinese Society of Gastroenterology IBD management guidelines]. Patient preference and shared decision making should be part of this process, more surveys such as the present study should be conducted to understand and then align patient and physician attitudes. Better incorporation of patient preferences in these pathways along with identifying knowledge gaps will subsequently lead to better education of both patients and physicians about performance characteristics of currently available therapies.

\section{Acknowledgments}

Funding: This research was funded by the National Natural Science Foundation of China $(81772650,81322037$, 81572302, 81421003), and the National Excellent Doctoral Dissertation of China (201182) to Dr. JL.

\section{Footnote}

Reporting Checklist: The authors have completed the SURGE reporting checklist. Available at https://dx.doi. org/10.21037/atm-21-3683

Data Sharing Statement: Available at https://dx.doi. org/10.21037/atm-21-3683

Conflicts of Interest: All authors have completed the ICMJE uniform disclosure form (available at https://dx.doi. 
org/10.21037/atm-21-3683). The authors have no conflicts of interest to declare.

Ethical Statement: The authors are accountable for all aspects of the work in ensuring that questions related to the accuracy or integrity of any part of the work are appropriately investigated and resolved. The study was conducted in accordance with the Declaration of Helsinki (as revised in 2013). The study was approved by the China Ethics Committee of Registering Clinical Trials (ChiECRCT20200202) and informed consent was taken from all the subjects.

Open Access Statement: This is an Open Access article distributed in accordance with the Creative Commons Attribution-NonCommercial-NoDerivs 4.0 International License (CC BY-NC-ND 4.0), which permits the noncommercial replication and distribution of the article with the strict proviso that no changes or edits are made and the original work is properly cited (including links to both the formal publication through the relevant DOI and the license). See: https://creativecommons.org/licenses/by-nc-nd/4.0/.

\section{References}

1. Cammarota G, Ianiro G, Tilg H, et al. European consensus conference on faecal microbiota transplantation in clinical practice. Gut 2017;66:569-80.

2. Kellermayer R. Fecal microbiota transplantation: great potential with many challenges. Transl Gastroenterol Hepatol 2019;4:40.

3. van Nood E, Vrieze A, Nieuwdorp M, et al. Duodenal infusion of donor feces for recurrent Clostridium difficile. N Engl J Med 2013;368:407-15.

4. Kassam Z, Lee CH, Yuan Y, et al. Fecal microbiota transplantation for Clostridium difficile infection: systematic review and meta-analysis. Am J Gastroenterol 2013;108:500-8.

5. Allegretti JR, Kassam Z, Mullish BH, et al. Effects of Fecal Microbiota Transplantation With Oral Capsules in Obese Patients. Clin Gastroenterol Hepatol 2020;18:855-863.e2.

6. Zhang Z, Mocanu V, Cai C, et al. Impact of Fecal Microbiota Transplantation on Obesity and Metabolic Syndrome-A Systematic Review. Nutrients 2019;11:2291.

7. Hartstra AV, Bouter KE, Bäckhed F, et al. Insights into the role of the microbiome in obesity and type 2 diabetes. Diabetes Care 2015;38:159-65.

8. D'Haens GR, Jobin C. Fecal Microbial Transplantation for Diseases Beyond Recurrent Clostridium Difficile Infection. Gastroenterology 2019;157:624-36.

9. Li Q, Han Y, Dy ABC, et al. The Gut Microbiota and Autism Spectrum Disorders. Front Cell Neurosci 2017;11:120.

10. Neurath MF. Current and emerging therapeutic targets for IBD. Nat Rev Gastroenterol Hepatol 2017;14:269-78.

11. Ren RR, Sun G, Yang YS, et al. Chinese physicians' perceptions of fecal microbiota transplantation. World J Gastroenterol 2016;22:4757-65.

12. Paramsothy S, Walsh AJ, Borody T, et al. Gastroenterologist perceptions of faecal microbiota transplantation. World J Gastroenterol 2015;21:10907-14.

13. Borody TJ, Khoruts A. Fecal microbiota transplantation and emerging applications. Nat Rev Gastroenterol Hepatol 2011;9:88-96.

14. Imdad A, Nicholson MR, Tanner-Smith EE, et al. Fecal transplantation for treatment of inflammatory bowel disease. Cochrane Database Syst Rev 2018;11:CD012774.

15. Rossen NG, Fuentes S, van der Spek MJ, et al. Findings From a Randomized Controlled Trial of Fecal Transplantation for Patients With Ulcerative Colitis. Gastroenterology 2015;149:110-8.e4.

16. Moayyedi P, Surette MG, Kim PT, et al. Fecal Microbiota Transplantation Induces Remission in Patients With Active Ulcerative Colitis in a Randomized Controlled Trial. Gastroenterology 2015;149:102-109.e6.

17. Zeitz J, Bissig M, Barthel C, et al. Patients' views on fecal microbiota transplantation: an acceptable therapeutic option in inflammatory bowel disease? Eur J Gastroenterol Hepatol 2017;29:322-30.

18. Kahn SA, Vachon A, Rodriquez D, et al. Patient perceptions of fecal microbiota transplantation for ulcerative colitis. Inflamm Bowel Dis 2013;19:1506-13.

19. Xu L, Zhang T, Cui B, et al. Clinical efficacy maintains patients' positive attitudes toward fecal microbiota transplantation. Medicine (Baltimore) 2016;95:e4055.

20. Zipursky JS, Sidorsky TI, Freedman CA, et al. Patient attitudes toward the use of fecal microbiota transplantation in the treatment of recurrent Clostridium difficile infection. Clin Infect Dis 2012;55:1652-8.

21. Paramsothy S, Kamm MA, Kaakoush NO, et al. Multidonor intensive faecal microbiota transplantation for active ulcerative colitis: a randomised placebo-controlled trial. Lancet 2017;389:1218-28.

22. Costello SP, Hughes PA, Waters O, et al. Effect of Fecal Microbiota Transplantation on 8-Week Remission in Patients With Ulcerative Colitis: A Randomized Clinical 
Trial. JAMA 2019;321:156-64.

23. Allegretti JR, Mullish BH, Kelly C, et al. The evolution of the use of faecal microbiota transplantation and emerging therapeutic indications. Lancet 2019;394:420-31.

24. Lane ER, Zisman TL, Suskind DL. The microbiota in inflammatory bowel disease: current and therapeutic insights. J Inflamm Res 2017;10:63-73.

25. Michail S, Durbin M, Turner D, et al. Alterations in the gut microbiome of children with severe ulcerative colitis. Inflamm Bowel Dis 2012;18:1799-808.

26. Akutko K, Stawarski A. Probiotics, Prebiotics and Synbiotics in Inflammatory Bowel Diseases. J Clin Med 2021;10:2466.

27. Vaughn BP, Rank KM, Khoruts A. Fecal Microbiota Transplantation: Current Status in Treatment of GI and Liver Disease. Clin Gastroenterol Hepatol 2019;17:353-61.

28. Wu X, Dai M, Buch H, et al. The recognition and attitudes of postgraduate medical students toward fecal microbiota transplantation: a questionnaire study. Therap Adv Gastroenterol 2019;12:1756284819869144.

29. Paramsothy S, Paramsothy R, Rubin DT, et al. Faecal Microbiota Transplantation for Inflammatory Bowel Disease: A Systematic Review and Meta-analysis. J Crohns Colitis 2017;11:1180-99.

30. Cui B, Feng Q, Wang H, et al. Fecal microbiota transplantation through mid-gut for refractory Crohn's disease: safety, feasibility, and efficacy trial results. J Gastroenterol Hepatol 2015;30:51-8.

Cite this article as: Zhang Y, Xue X, Su S, Zhou H, Jin Y, Shi Y, Lin J, Wang J, Li X, Yang G, Philpott JR, Liang J. Patients and physicians' attitudes change on fecal microbiota transplantation for inflammatory bowel disease over the past 3 years. Ann Transl Med 2021;9(21):1619. doi: 10.21037/atm-21-3683
31. Suskind DL, Brittnacher MJ, Wahbeh G, et al. Fecal microbial transplant effect on clinical outcomes and fecal microbiome in active Crohn's disease. Inflamm Bowel Dis 2015;21:556-63.

32. He Z, Li P, Zhu J, et al. Multiple fresh fecal microbiota transplants induces and maintains clinical remission in Crohn's disease complicated with inflammatory mass. Sci Rep 2017;7:4753.

33. Peng Z, Xiang J, He Z, et al. Colonic transendoscopic enteral tubing: A novel way of transplanting fecal microbiota. Endosc Int Open 2016;4:E610-3.

34. Cui B, Li P, Xu L, et al. Step-up fecal microbiota transplantation (FMT) strategy. Gut Microbes 2016;7:323-8.

35. König J, Siebenhaar A, Högenauer C, et al. Consensus report: faecal microbiota transfer - clinical applications and procedures. Aliment Pharmacol Ther 2017;45:222-39.

36. Qazi T, Amaratunga T, Barnes EL, et al. The risk of inflammatory bowel disease flares after fecal microbiota transplantation: Systematic review and meta-analysis. Gut Microbes 2017;8:574-88.

37. Fischer M, Kao D, Kelly C, et al. Fecal Microbiota Transplantation is Safe and Efficacious for Recurrent or Refractory Clostridium difficile Infection in Patients with Inflammatory Bowel Disease. Inflamm Bowel Dis 2016;22:2402-9.

(English Language Editor: J. Jones) 